\title{
Safety and Efficacy of Low-Dose Doxorubicin and Fluorouracil (5FU) with Best Supportive Care Compared To Best Supportive Care Alone for Patients with Advanced Hepatocellular Carcinoma
}

\author{
Bader A. Abdelmaksoud*, Mostafa M. Toam, Alaa A. Fayed \\ Department of clinical oncology and nuclear medicine, Faculty of Medicine, Zagazig university, Zagazig, Egypt
}

*Corresponding author: Bader A. Abdelmaksoud, Department of clinical oncology and nuclear medicine, Faculty of Medicine, Zagazig university, Zagazig, Egypt, E-mail: bader6a@yahoo.com

\begin{abstract}
Aim:To evaluate the safety and efficacy of low-dose doxorubicin and 5FU with best supportive care compared to best supportive care alone for patients with advanced hepatocellular carcinoma.

Patients and methods: Atotal of 60 patients ( 49 male and 11 females) with advanced hepatocellular carcinoma were enrolled. All patients were randomly divided into two groups. Treatment group: patients receive one day cycle of intravenous doxorubicin $20 \mathrm{mg} / \mathrm{m}^{2}$ and 5FU $500 \mathrm{mg}$ along with best supportive care, the cycle repeated every two weeks continuously until disease progression, unacceptable toxicity or patient refusal. Control group (best supportive care only): Patients received supportive treatment in the form of liver support, tonics, and other symptomatic treatment until death or patient refusal.

Results: After a median follow- up of one year, all patients died except three patients still alive. There were 5 patients $(16.7 \%$ ) in treatment group (group A) achieved Partial Response(PR) compared to No Response (NR) in all patients (100\%) of control group (group B )(P -value 0.052). the median Progression Free Survival (PFS) was 5 months in group A and 3.5 months in group B, ( P- value 0.018 ), also, in group A the median Overall Survival (OS) was 8 months compared to 6 months in group B, with one year (OS) rates $9.4 \%$ and $3.7 \%$ in group A and B respectively (P-value 0.125 ).

there were minimal treatment-related toxicities, and all patients completed treatment without interruption.

Conclusion: Low dose doxorubicin with 5FU are well tolerated and shows modest anti- tumor efficacy in patients with advanced hepatocellular carcinoma.
\end{abstract} Received Date: October 31, 2016 Accepted Date: January 06, 2017 Published Date: January 13, 2017

Citation: Abdelmaksoud, B.A., et al. Safety and Efficacy of Low-Dose Doxorubicin and Fluorouracil (5FU) with Best Supportive Care Compared To Best Supportive Care Alone for Patients with Advanced Hepatocellular Carcinoma. (2017) Int J Cancer Oncol 4(1): 178- 185.

DOI: $10.15436 / 2377-0902.17 .1194$

Keywords: Hepatocellular carcinoma; Low dose doxorubicin; 5FU

\section{Introduction}

Globally, Hepatocellular Carcinoma (HCC) is the fifth most common cancer of all malignancies and the third most common cause of cancer-related mortality in the world. In both developing and developed countries, the incidence of HCC has significantly increased over the recent decades ${ }^{[1,2]}$. In Egypt, liver is the commonest site of cancer in males (18.7\%) and the third most common site in females $(4.6 \%)^{[3]}$. Development of HCC generally occurs due to different underlying risk factors e.g. [cirrhosis, hepatitis C and B viruses, hemochromatosis, Wilson's disease, biliary cirrhosis, and other abnormal liver conditions $]^{[4]}$ . After diagnosis of HCC, surgery such as surgical resection or transplantation is considered a potentially curative method for HCC treatment, but it is only applicable to small proportion of patients, for patients with localized unresectable HCC, localized therapy, such as percutaneous thermo-ablation or transarterial chemoembolization, has been reported to be useful for treating such patients, but in most cases, the disease recurs or progresses to an advanced stage for which local treatments are in effective 
or not applicable, therefore, these groups of patients are in need for systemic therapy ${ }^{[5,6]}$. For patients presenting with locally advanced or metastatic HCC, there is no approved systemic treatment except sorafenib ${ }^{[7]}$. The role of systemic chemotherapy for advanced or metastatic HCC has not been established despite numerous chemotherapeutic agents have been investigated ${ }^{[8]}$. Results of prospective phase II/III clinical trials to investigate the efficacy of doxorubicin in advanced HCC showed that doxorubicin can be effective in about $20 \%$ of cases when used as single agent but without Overall Survival (OS) advantages ${ }^{[9-12]}$.

Fluorouracil (5-FU) was also commonly used and had undergone extensive evaluation in hepatocellular carcinoma with response rates around $10 \%$ in many phase II clinical studies $^{[13-15]}$. Regarding to combination chemotherapy, until now no combination has been proven to have higher activity compared to single agents ${ }^{[16]}$. Based on the results of randomized trials and retrospective studies that tested the role of doxorubicin and 5FU in treatment of advanced HCC, we conducted this study to evaluate the safety and efficacy of low-dose doxorubicin when combined with 5FU along with supportive measures compared with best standard of care for cases had advanced HCC not suitable for curative local therapies.

\section{Patients and Methods}

\section{Patients eligibility}

Patients had hepatocellular carcinoma confirmed by biopsy, typical radiological criteria applicable in HCC and/or level of alpha fetoprotein above $200 \mathrm{ng} / \mathrm{ml}$ were enrolled. Regarding staging, Patients had either distant metastases or non metastatic locally advanced disease not suitable for other therapeutic modalities involved in management of HCC e.g chemoembolization and sorafenib due to any cause. Other inclusion criteria were age $>18$ years, ECOG performance status $\leq 2$, adequat liver reserve [Child-Pugh score of A], bilirubin $2.0 \mathrm{mg} / \mathrm{dL}$ or less, transaminases level 4 times or lower than upper limit of normal (ULN)], adequate renal and bone marrow function specifically serum creatinine level $2.0 \mathrm{mg} / \mathrm{dL}$ or less, platelets $\geq 100,000 /$ $\mathrm{mm}^{3}$, neutrophil count $\geq 1000 / \mathrm{mm}^{3}$, hemoglobin $\geq 10 \mathrm{~g} / \mathrm{dL}$. The main exlusion criteria include Child-Pugh class B or $\mathrm{C}$, chronic active hepatitis B not treated, and/or patients suitable for curative local treatments measures ( liver transplantation, resection, percutaneous ablative therapy), poor cardiac reserve( $\mathrm{EF}<60 \%$ ) and other malignancies in the body elsewhere. Written informed consent was taken.

\section{Treatment schedule}

For patients met the above inclusion criteria, they were randomly divided into two groups. Experimental group: patients receive intravenous doxorubicin $20 \mathrm{mg} / \mathrm{m}^{2}$ and $5 \mathrm{FU} 500 \mathrm{mg}$, one day cycle. The cycle repeated every two weeks continuously until disease progression, unacceptable toxicity or patient refusal. In between chemotherapy cycles, patients were maintained on supportive treatment also. Control group (best supportive care only): Patients received supportive treatment in the form of liver supports, tonics, and other symptomatic treatment until death or patient refusal. Before starting treatment, patients were subjected to full medical history and physical examination, performance status assessment, evidence of recent weight loss and other comorbidities as cardiac diseases. Other studies included a full and differential blood count, kidney and liver function tests, Alpha Feto Protien (AFP), pelvi- abdomial CT scan,and chest $\mathrm{X}$ - ray.

\section{Treatment evaluation and follow- up}

The patients were monitored during treatment every week by physical examination for determination of patient's compliance to treatment and possible side effects. A complete blood picture, kidney, and liver functions were considered prior to every cycle. Patients received four cycles ( 8 weeks) were well thought-out for evaluation of response by triphasic CT and AFP continuously during treatment. Response assessment was done according to revised RECIST guideline as follow: Complete Response (CR) was defined as complete disappearance of all radiological and clinical evidence of tumor. Partial Response (PR) was defined as $30 \%$ decrease in the sum of diameters of target lesions. Progressive Disease (PD) was considered if there was appearance of new lesions, there was an increase in the size of the tumor size by $\geq 25 \%$ compared to pretreatment size or if there were deterioration in the patient clinical conditions due to disease progression. Stable Disease (SD) was considered if the patient not met criteria of CR, PR or PD mentioned above and remained for at least 2 cycles of treatment. The toxicity profile was based on the NCI commontoxicity criteria.

\section{Endpoints}

The primary endpoints of this study were overall survival and safety profile. Secondary endpoints were response and Progression Free Survival (PFS) rates.

\section{Statistical analysis}

Continuous variables were expressed as the mean \pm SD $\&$ median (range), and the categorical variables were expressed as number (percentage). Comparison between the two groups of normally distributed variables was done using independent samples Student's t-tests, while Mann Whitney U test was used for non-normally distributed variables. Overall Survival (OS) was calculated as the time from randomization to death or the most recent follow-up contact (censored) but Progression Free Survival (PFS)/Time to Tumor Progression (TTP) was calculated as the time from randomization to tumor progression. Stratification of OS and PFS was done according to all basic characteristics and response to treatment. These time-to-event distributions were estimated using the method of Kaplan-Meier plot, and compared using two-sided exact log-rank test. All tests were- two sided. A p-value of $<0.05$ was considered significant.

\section{Results}

\section{Basic characteristics}

A total of 60 patients with advanced HCC were enrolled, 30 patients in each group. In the two groups, the demographic basic characteristics were well balanced as possible. The clinicodemographic parameters and treatment outcome are shown in (Table 1). Regarding the age, the median age was 51.5, range (39 to 62 years) in group A and 53.5, ranging from 41 to 65 years in group B. The study includes 49 males, 25 in group A and 24 in group B, and 11 females, 5 in group A and 6 in group B. Regarding ECOG PS, group A includes, 9 patients (30\%) had PS of 1 and 21 patients(70\%) had PS of 2, while group B includes, 7 
patients (23.3\%) had PS of 1 and 23 patients (66.7\%) had PS of 2. Of patients in group A, $14(46.7 \%)$ had no previous treatment, $6(20 \%)$ treated with PEI, 3(10\%) underwent RFA, 5(16.7\%) underwent TACE, and $2(6.7 \%)$ had combination of RFA and TACE, while in group B, $16(53.3 \%)$ had no previous treatment, $4(13.3 \%)$ treated with Percutaneous Ethanol Injection(PEI), 4 (13.3\%) underwent Radio Frequency Ablation (RFA), 4 (13.3\%) under went Transarterial Chemoembolization(TACE), and 2 $(6,7 \%)$ had combination of RFA and TACE. All patients in this study were Child - Pugh class A, but some patients were either of score 5 or 6 in both groups, in group A, there were 20 patients $(66.6 \%)$ with score 5 and $10(33.3 .3 \%)$ score 6 , while in group B, there were 11(36.7\%) with score 5 and 19(63.3\%) score 6 .

Table 1: Basic Characteristics and Treatment Outcome in both Experimental Group (Group A) and Control Group (Group B) .

\begin{tabular}{|c|c|c|c|c|c|}
\hline \multirow{2}{*}{ Characteristics } & \multicolumn{2}{|c|}{$\operatorname{Group} A(N=30)$} & \multicolumn{2}{|c|}{ Group B $(\mathbf{N}=\mathbf{3 0})$} & \multirow{2}{*}{ p-value } \\
\hline & No. & $(\%)$ & No. & $(\%)$ & \\
\hline \multicolumn{6}{|l|}{ Age (years) } \\
\hline Mean \pm SD & 51.66 & \pm 6.60 & 52.96 & \pm 7.08 & \multirow{2}{*}{$0.465^{*}$} \\
\hline Median (Range) & 51.50 & $(39-62)$ & 53.50 & $(41-65)$ & \\
\hline$\leq 40$ years & 2 & $(6.7 \%)$ & 0 & $(0 \%)$ & \multirow{3}{*}{$0.242+$} \\
\hline 41 - 59 years & 24 & $(80 \%)$ & 23 & $(76.7 \%)$ & \\
\hline$\geq 60$ years & 4 & $(13.3 \%)$ & 7 & $(23.3 \%)$ & \\
\hline \multicolumn{6}{|l|}{ Sex } \\
\hline Male & 25 & $(83.3 \%)$ & 24 & $(80 \%)$ & \multirow{2}{*}{$0.739 \ddagger$} \\
\hline Female & 5 & $(16.7 \%)$ & 6 & $(20 \%)$ & \\
\hline \multicolumn{6}{|l|}{ ECOG PS } \\
\hline ECOG 1 & 9 & $(30 \%)$ & 7 & $(23.3 \%)$ & \multirow{2}{*}{$0.559+$} \\
\hline ECOG 2 & 21 & $(70 \%)$ & 23 & $(76.7 \%)$ & \\
\hline \multicolumn{6}{|c|}{ Previous treatment } \\
\hline No & 14 & $(46.7 \%)$ & 16 & $(53.3 \%)$ & \multirow{5}{*}{$0.940 \%$} \\
\hline PEI & 6 & $(20 \%)$ & 4 & $(13.3 \%)$ & \\
\hline RFA & 3 & $(10 \%)$ & 4 & $(13.3 \%)$ & \\
\hline TACE & 5 & $(16.7 \%)$ & 4 & $(13.3 \%)$ & \\
\hline RFA+TACE & 2 & $(6.7 \%)$ & 2 & $(6.7 \%)$ & \\
\hline \multicolumn{6}{|l|}{ Child score } \\
\hline Score 5 & 20 & $(66.7 \%)$ & 11 & $(36.7 \%)$ & \multirow{2}{*}{$0.020 \ddagger$} \\
\hline Score 6 & 10 & $(33.3 \%)$ & 19 & $(63.3 \%)$ & \\
\hline \multicolumn{6}{|l|}{ Response } \\
\hline NR & 25 & $(83.3 \%)$ & 30 & $(100 \%)$ & \multirow{2}{*}{$0.052 \ddagger$} \\
\hline OAR & 5 & $(16.7 \%)$ & 0 & $(0 \%)$ & \\
\hline PD & 9 & $(30 \%)$ & 19 & $(63.3 \%)$ & \multirow{3}{*}{$0.009 \ddagger$} \\
\hline SD & 16 & $(53.3 \%)$ & 11 & $(36.7 \%)$ & \\
\hline PR & 5 & $(16.7 \%)$ & 0 & $(0 \%)$ & \\
\hline \multicolumn{6}{|l|}{ PFS } \\
\hline Median PFS & \multicolumn{2}{|c|}{5 months } & \multicolumn{2}{|c|}{3.5 months } & \multirow{2}{*}{$0.018 \S$} \\
\hline 6 mon PFS & & & & & \\
\hline \multicolumn{6}{|l|}{ OS } \\
\hline Median OS & \multicolumn{2}{|c|}{8 months } & \multicolumn{2}{|c|}{6 months } & \multirow{3}{*}{$0.125 \S$} \\
\hline 6 mon OS & \multicolumn{2}{|c|}{$73.3 \%$} & \multicolumn{2}{|c|}{$40 \%$} & \\
\hline 12 mon OS & & & & & \\
\hline
\end{tabular}

* Independent samples Student's t-test,

†Chi-square test, § Chi-square test for trend.

$\mathrm{P}<0.05$ is significant.

\section{Treatment outcome}

After median follow- up of 12 months range (6 - 18), all patients died except, three patients still alive, two in group A and 
one in group B. Regarding to tumor response in the two groups, there were five patients $(16.7 \%)$ in group A achieved overall response (OAR) in the form of partial response(PR) compared to no response $(\mathrm{NR})$ in all patients $(100 \%)$ of group B (P-value < $0.052)$, also, there were nine patients $(30 \%)$ in group A showed progressive disease (PD) compared to 19 patients $(63.3 \%)$ in group B, and 16 patients $(53.3 \%)$ with stable disease in group A compared to 11 patients $(36.7 \%)$ in group B (P-value 0.009$)$.
In this study, the median progression free survival (PFS) was 5 months in group A and 3.5 months in group B, with 6 months PFS rate of $10 \%$ and $3.3 \%$ in group $A$ and $B$ respectively ( $\mathrm{P}$ - value 0.018 ), also, in group A the median overall survival (OS) was 8 months compared to 6 months in group B, with one year (OS) rate of $9.4 \%$ and $3.7 \%$ in group $A$ and $B$ respectively (P-value0.125), (figure 1).

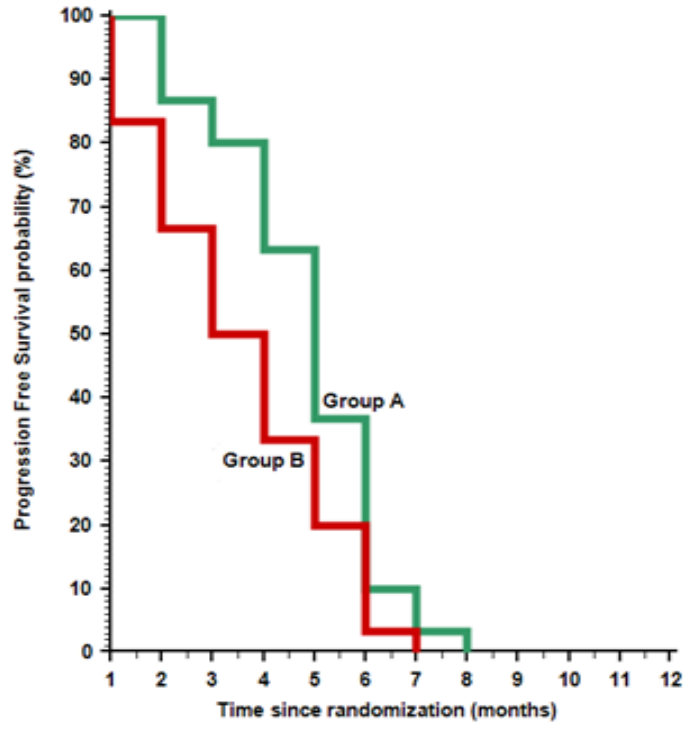

(A)

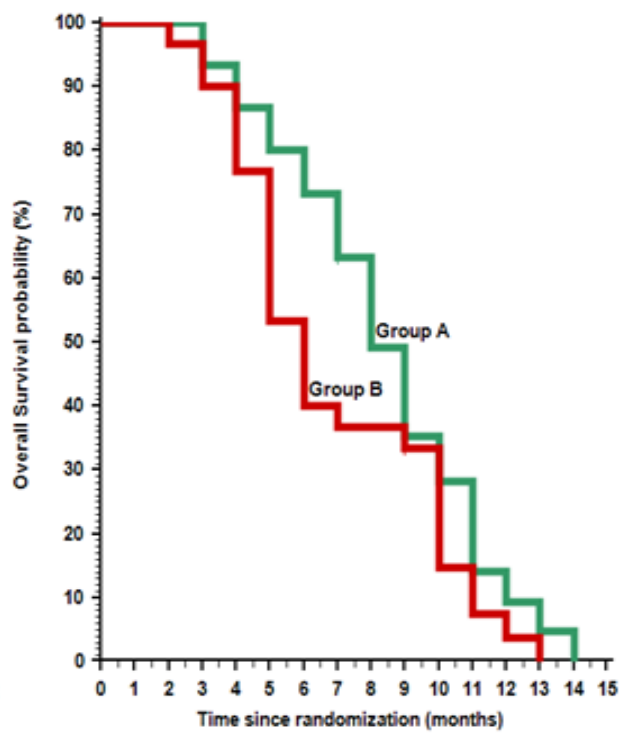

(B)

Figure 1: Kaplan-Meier plot: (A) Progression Free Survival (PFS); (B) Overall survival (OS).

Table 2: Effect of Basic Characteristics on Response to Treatment in 30 Patients with HCC (Group A).

\begin{tabular}{|c|c|c|c|c|c|c|c|c|c|c|c|c|c|c|}
\hline \multirow{3}{*}{$\begin{array}{l}\text { Charac- } \\
\text { teristics }\end{array}$} & & & \multicolumn{4}{|c|}{ Response } & \multirow{3}{*}{$\begin{array}{c}\text { p- } \\
\text { value }\end{array}$} & \multicolumn{6}{|c|}{ Response } & \multirow{3}{*}{$\begin{array}{c}\text { p- } \\
\text { value }\end{array}$} \\
\hline & \multicolumn{2}{|c|}{$\begin{array}{c}\text { All } \\
(\mathbf{N}=\mathbf{3 0})\end{array}$} & \multicolumn{2}{|c|}{$\begin{array}{c}\text { NR } \\
(N=25)\end{array}$} & \multicolumn{2}{|c|}{$\begin{array}{c}\text { OAR } \\
(\mathrm{N}=5)\end{array}$} & & \multicolumn{2}{|c|}{ PD $(N=9)$} & \multicolumn{2}{|c|}{$\operatorname{SD}(N=16)$} & \multicolumn{2}{|c|}{$\operatorname{PR}(\mathrm{N}=5)$} & \\
\hline & No. & $(\%)$ & No. & $(\%)$ & No. & $(\%)$ & & No. & $(\%)$ & No. & $(\%)$ & No. & $(\%)$ & \\
\hline \multicolumn{15}{|c|}{ Age (years) } \\
\hline $\begin{array}{l}\text { Mean } \pm \\
\text { SD }\end{array}$ & 51.66 & \pm 6.60 & 51.96 & \pm 6.37 & 50.20 & \pm 8.31 & \multirow{2}{*}{$0.595^{*}$} & 53.44 & \pm 7.24 & 51.12 & \pm 5.90 & 50.20 & \pm 8.31 & \multirow{2}{*}{$0.621 *$} \\
\hline $\begin{array}{l}\text { Median } \\
\text { (Range) }\end{array}$ & 51.50 & $(39-62)$ & 52 & $(40-62)$ & 51 & $(39-59)$ & & 54 & $(40-62)$ & 51.50 & $(41-62)$ & 51 & $(39-59)$ & \\
\hline $\begin{array}{l}\leq 40 \\
\text { years }\end{array}$ & 2 & $(6.7 \%)$ & 1 & $(50 \%)$ & 1 & $(50 \%)$ & \multirow{3}{*}{$0.301 \ddagger$} & 1 & $(50 \%)$ & 0 & $(0 \%)$ & 1 & $(50 \%)$ & \multirow{3}{*}{$0.104 \$$} \\
\hline $\begin{array}{l}41-59 \\
\text { years }\end{array}$ & 24 & $(80 \%)$ & 20 & $(83.3 \%)$ & 4 & $(16.7 \%)$ & & 5 & $(20.8 \%)$ & 15 & $(62.5 \%)$ & 4 & $(16.7 \%)$ & \\
\hline $\begin{array}{l}\geq 60 \\
\text { years }\end{array}$ & 4 & $(13.3 \%)$ & 4 & $(100 \%)$ & 0 & $(0 \%)$ & & 3 & $(75 \%)$ & 1 & $(25 \%)$ & 0 & $(0 \%)$ & \\
\hline \multicolumn{15}{|l|}{ Sex } \\
\hline Male & 25 & $(83.3 \%)$ & 21 & $(84 \%)$ & 4 & $(16 \%)$ & \multirow{2}{*}{$1.000 t$} & 8 & $(32 \%)$ & 13 & $(52 \%)$ & 4 & $(16 \%)$ & \multirow{2}{*}{$0.865 t$} \\
\hline Female & 5 & $(16.7 \%)$ & 4 & $(80 \%)$ & 1 & $(20 \%)$ & & 1 & $(20 \%)$ & 3 & $(60 \%)$ & 1 & $(20 \%)$ & \\
\hline \multicolumn{15}{|c|}{ ECOG PS } \\
\hline ECOG 1 & 9 & $(30 \%)$ & 6 & $(66.7 \%)$ & 3 & $(33.3 \%)$ & \multirow{2}{*}{$0.143 \$$} & 2 & $(22.2 \%)$ & 4 & $(44.4 \%)$ & 3 & $(33.3 \%)$ & \multirow{2}{*}{0.274} \\
\hline ECOG 2 & 21 & $(70 \%)$ & 19 & $(90.5 \%)$ & 2 & $(9.5 \%)$ & & 7 & $(33.3 \%)$ & 12 & $(57.1 \%)$ & 2 & $(9.5 \%)$ & \\
\hline \multicolumn{15}{|c|}{ Previous treatment } \\
\hline No & 14 & $(46.7 \%)$ & 10 & $(71.4 \%)$ & 4 & $(28.6 \%)$ & \multirow{3}{*}{$0.489 \ddagger$} & 2 & $(14.3 \%)$ & 8 & $(57.1 \%)$ & 4 & $(28.6 \%)$ & \multirow{3}{*}{0.633} \\
\hline PEI & 6 & $(20 \%)$ & 5 & $(83.3 \%)$ & 1 & $(16.7 \%)$ & & 2 & $(33.3 \%)$ & 3 & $(50 \%)$ & 1 & $(16.7 \%)$ & \\
\hline RFA & 3 & $(10 \%)$ & 3 & $(100 \%)$ & 0 & $(0 \%)$ & & 1 & $(33.3 \%)$ & 2 & $(66.7 \%)$ & 0 & $(0 \%)$ & \\
\hline
\end{tabular}


Safety and Efficacy of Low-Dose Doxorubicin and Fluorouracil (5FU)

\begin{tabular}{|l|c|c|c|c|c|c|c|c|c|c|c|c|c|c|c|c|}
\hline \hline TACE & 5 & $(16.7 \%)$ & 5 & $(100 \%)$ & 0 & $(0 \%)$ & & 3 & $(60 \%)$ & 2 & $(40 \%)$ & 0 & $(0 \%)$ & \\
\cline { 1 - 1 } $\begin{array}{l}\text { RFA+- } \\
\text { TACE }\end{array}$ & 2 & $(6.7 \%)$ & 2 & $(100 \%)$ & 0 & $(0 \%)$ & & 1 & $(50 \%)$ & 1 & $(50 \%)$ & 0 & $(0 \%)$ & \\
\hline Child score & & &
\end{tabular}

* Independent samples Student's t-test for two groups \& One way ANOVA test for more than 2 groups;

\$Chi-square test;

$\mathrm{p}<0.05$ is significant.

In this study, the effect of patient basic characteristics on response to treatment and subsequently the effect of both on progression free survival and overall survival were statistically analyzed into group A to determine the impact of variable prognostic factors on treatment outcome (Tables 2,3,4). From this subset analysis; previous treatments, Child score, and treatment response were the prognostic factors that have a significant impact on PFS but not on OS, while other factors as age, sex, and PS showed no significant impact on both PFS or OS.

Table 3: Effect of Basic Characteristics and Response to Treatment on Progression Free Survival in 30 Patients with HCC (Group A).

\begin{tabular}{|c|c|c|c|c|c|c|}
\hline \multirow{3}{*}{ Characteristics } & & & \multicolumn{3}{|c|}{ Progression Free Survival (PFS) } & \multirow{3}{*}{ p-value } \\
\hline & \multicolumn{2}{|c|}{ All $(\mathbf{N}=\mathbf{3 0})$} & \multirow{2}{*}{$\begin{array}{l}\text { Median TTP } \\
\text { (months) }\end{array}$} & \multirow{2}{*}{$\begin{array}{c}3 \text { month PFS } \\
(\%)\end{array}$} & \multirow{2}{*}{$\begin{array}{c}6 \text { month PFS } \\
(\%)\end{array}$} & \\
\hline & No. & $(\%)$ & & & & \\
\hline All patients & 30 & $(100 \%)$ & 5 months & $80 \%$ & $10 \%$ & \\
\hline \multicolumn{7}{|l|}{ Age (years) } \\
\hline$\leq 40$ years & 2 & $(6.7 \%)$ & 5 months & $100 \%$ & $50 \%$ & \multirow{3}{*}{$0.369 \dagger$} \\
\hline 41-59 years & 24 & $(80 \%)$ & 5 months & $79.2 \%$ & $8.3 \%$ & \\
\hline$\geq 60$ years & 4 & $(13.3 \%)$ & 4 months & $75 \%$ & $0 \%$ & \\
\hline \multicolumn{7}{|l|}{ Sex } \\
\hline Male & 25 & $(83.3 \%)$ & 5 months & $76 \%$ & $12 \%$ & \multirow{2}{*}{$0.417 \dagger$} \\
\hline Female & 5 & $(16.7 \%)$ & 6 months & $100 \%$ & $0 \%$ & \\
\hline \multicolumn{7}{|l|}{ ECOG PS } \\
\hline ECOG 1 & 9 & $(30 \%)$ & 5 months & $88.9 \%$ & $22.2 \%$ & \multirow{2}{*}{$0.567 \dagger$} \\
\hline ECOG 2 & 21 & $(70 \%)$ & 5 months & $76.1 \%$ & $4.7 \%$ & \\
\hline \multicolumn{7}{|c|}{ Previous treatment } \\
\hline No & 14 & $(46.7 \%)$ & 6 months & $92.9 \%$ & $7.1 \%$ & \multirow{5}{*}{$0.009 \dagger$} \\
\hline PEI & 6 & $(20 \%)$ & 5 months & $100 \%$ & $33.3 \%$ & \\
\hline RFA & 3 & $(10 \%)$ & 5 months & $100 \%$ & $0 \%$ & \\
\hline TACE & 5 & $(16.7 \%)$ & 5 months & $60 \%$ & $0 \%$ & \\
\hline RFA+TACE & 2 & $(6.7 \%)$ & 2 months & $0 \%$ & $0 \%$ & \\
\hline \multicolumn{7}{|l|}{ Child score } \\
\hline Score 5 & 20 & $(66.7 \%)$ & 5 months & $100 \%$ & $15 \%$ & \multirow{2}{*}{$<0.001 \dagger$} \\
\hline Score 6 & 10 & $(33.3 \%)$ & 3 months & $40 \%$ & $0 \%$ & \\
\hline \multicolumn{7}{|l|}{ Response } \\
\hline NR & 25 & $(83.3 \%)$ & 5 months & $84 \%$ & $28 \%$ & \multirow{2}{*}{$0.021 \dagger$} \\
\hline OAR & 5 & $(16.7 \%)$ & 6 months & $100 \%$ & $80 \%$ & \\
\hline PD & 9 & $(30 \%)$ & 4 months & $55.6 \%$ & $0 \%$ & \multirow{3}{*}{$0.006 \dagger$} \\
\hline SD & 16 & $(53.3 \%)$ & 5 months & $87.5 \%$ & $37.5 \%$ & \\
\hline PR & 5 & $(16.7 \%)$ & 6 months & $100 \%$ & $80 \%$ & \\
\hline
\end{tabular}

NR denote not reached,

$\dagger$ Log rank test,

$\mathrm{p}<0.05$ is significant. 
Safety and Efficacy of Low-Dose Doxorubicin and Fluorouracil (5FU)

Table 4: Effect of Basic Characteristics and Response to Treatment on Overall Survival in 30 patients with HCC (Group A).

\begin{tabular}{|c|c|c|c|c|c|c|c|c|c|c|c|}
\hline \multirow{4}{*}{$\begin{array}{l}\text { Characteristics } \\
\text { All patients }\end{array}$} & & & \multicolumn{4}{|c|}{ Survival } & \multirow{4}{*}{ p-value } & \multicolumn{3}{|c|}{ Overall Survival (OS) } & \multirow{4}{*}{ p-value } \\
\hline & \multicolumn{2}{|c|}{ All $(N=30)$} & \multicolumn{2}{|c|}{ Alive $(\mathrm{N}=2)$} & \multicolumn{2}{|c|}{$\operatorname{Died}(\mathrm{N}=28)$} & & \multirow{3}{*}{\begin{tabular}{|c|}
$\begin{array}{l}\text { Median OS } \\
\text { (months) }\end{array}$ \\
8 months
\end{tabular}} & \multirow{3}{*}{$\begin{array}{c}\text { month } \\
\text { OS (\%) } \\
93.3 \% \\
\end{array}$} & \multirow{3}{*}{$\begin{array}{c}6 \text { month } \\
\text { OS (\%) } \\
73.3 \%\end{array}$} & \\
\hline & No. & $(\%)$ & No. & $(\%)$ & No. & $(\%)$ & & & & & \\
\hline & 30 & $(100 \%)$ & 2 & $(6.7 \%)$ & 28 & $(93.3 \%)$ & & & & & \\
\hline \multicolumn{12}{|l|}{ Age (years) } \\
\hline Mean \pm SD & 51.66 & \pm 6.60 & 50.50 & \pm 7.77 & 51.75 & \pm 6.67 & \multirow{2}{*}{$0.803 \bullet$} & & & & \\
\hline Median (Range) & 51.50 & $(39-62)$ & 50.50 & $(45-56)$ & 51.50 & $(39-62)$ & & & & & \\
\hline$\leq 40$ years & 2 & $(6.7 \%)$ & 0 & $(0 \%)$ & 2 & $(100 \%)$ & \multirow{3}{*}{$0.765 \ddagger$} & 10 months & $100 \%$ & $100 \%$ & \multirow{3}{*}{$0.504 \uparrow$} \\
\hline 41-59 years & 24 & $(80 \%)$ & 2 & $(8.3 \%)$ & 22 & $(91.7 \%)$ & & 8 months & $91.7 \%$ & $75 \%$ & \\
\hline$\geq 60$ years & 4 & $(13.3 \%)$ & 0 & $(0 \%)$ & 4 & $(100 \%)$ & & 6 months & $100 \%$ & $50 \%$ & \\
\hline \multicolumn{12}{|l|}{ Sex } \\
\hline Male & 25 & $(83.3 \%)$ & 1 & $(4 \%)$ & 24 & $(96 \%)$ & \multirow{2}{*}{$0.310 \ddagger$} & 8 months & $92 \%$ & $68 \%$ & \multirow{2}{*}{$0.547 \dagger$} \\
\hline Female & 5 & $(16.7 \%)$ & 1 & $(20 \%)$ & 4 & $(80 \%)$ & & 9 months & $100 \%$ & $100 \%$ & \\
\hline \multicolumn{12}{|l|}{ ECOG PS } \\
\hline ECOG 1 & 9 & $(30 \%)$ & 0 & $(0 \%)$ & 9 & $(100 \%)$ & \multirow{2}{*}{$1.000 \ddagger$} & 8 months & $100 \%$ & $66.7 \%$ & \multirow{2}{*}{$0.910 \dagger$} \\
\hline ECOG 2 & 21 & $(70 \%)$ & 2 & $(9.5 \%)$ & 19 & $(90.5 \%)$ & & 9 months & $90.5 \%$ & $76.1 \%$ & \\
\hline \multicolumn{12}{|c|}{ Previous treatment } \\
\hline No & 14 & $(46.7 \%)$ & 2 & $(14.3 \%)$ & 12 & $(85.7 \%)$ & \multirow{5}{*}{$0.654 \ddagger$} & 9 months & $100 \%$ & $85.8 \%$ & \multirow{5}{*}{$<0.001 \uparrow$} \\
\hline PEI & 6 & $(20 \%)$ & 0 & $(0 \%)$ & 6 & $(100 \%)$ & & 8 months & $100 \%$ & $83.3 \%$ & \\
\hline RFA & 3 & $(10 \%)$ & 0 & $(0 \%)$ & 3 & $(100 \%)$ & & 7 months & $100 \%$ & $66.7 \%$ & \\
\hline TACE & 5 & $(16.7 \%)$ & 0 & $(0 \%)$ & 5 & $(100 \%)$ & & 9 months & $100 \%$ & $60 \%$ & \\
\hline RFA+TACE & 2 & $(6.7 \%)$ & 0 & $(0 \%)$ & 2 & $(100 \%)$ & & 3 months & $0 \%$ & $0 \%$ & \\
\hline \multicolumn{12}{|l|}{ Child score } \\
\hline Score 5 & 20 & $(66.7 \%)$ & 2 & $(10 \%)$ & 18 & $(90 \%)$ & \multirow{2}{*}{$0.540 \ddagger$} & 9 months & $100 \%$ & $80 \%$ & \multirow{2}{*}{$0.003 \dagger$} \\
\hline Score 6 & 10 & $(33.3 \%)$ & 0 & $(0 \%)$ & 10 & $(100 \%)$ & & 5 months & $90 \%$ & $40 \%$ & \\
\hline \multicolumn{12}{|l|}{ Response } \\
\hline NR & 25 & $(83.3 \%)$ & 2 & $(8 \%)$ & 23 & $(92 \%)$ & $1000+$ & 8 months & $100 \%$ & $76 \%$ & م0R2. \\
\hline OAR & 5 & $(16.7 \%)$ & 0 & $(0 \%)$ & 5 & $(100 \%)$ & $1.000_{4}^{+}$ & 11 months & $100 \%$ & $100 \%$ & 0.0021 \\
\hline PD & 9 & $(30 \%)$ & 1 & $(11.1 \%)$ & 8 & $(88.9 \%)$ & & 6 months & $88.9 \%$ & $44.4 \%$ & \\
\hline SD & 16 & $(53.3 \%)$ & 1 & $(6.3 \%)$ & 15 & $(93.8 \%)$ & $0.723 \ddagger$ & 8 months & $93.7 \%$ & $81.3 \%$ & $0.090 \dagger$ \\
\hline PR & 5 & $(16.7 \%)$ & 0 & $(0 \%)$ & 5 & $(100 \%)$ & & 11 months & $100 \%$ & $100 \%$ & \\
\hline
\end{tabular}

- Mann Whitney U test; †Chi-square test;

$\dagger$ Log rank test;

$\mathrm{p}<0.05$ is significant.

\section{Treatment toxcity}

Regarding haematological toxicities, there were no grade III \& IV toxicities. There was only grade I \& II anaemiain $(20 \%)$ which do not need cycle interruption. Other non-haematological toxicities include grade II \& III anorexia, vomiting and diarrhea (25\%), grade I \& II stomatitis (10\%), grade I \& II alopecia (5\%), grade I elevated liver enzymes $(30 \%)$, grade II \& III fatigue $(10 \%)$. There was no any treatment -related mortality observed in this study.

\section{Discussion}

Until now, there is no approved systemic treatment applicable for patients with advanced HCC except sorafenib. Although, sorafenib is the only systemic treatment demonstrating significant statistically but modest overall survival advantages in phase III randomized, placebo-controlled trial, it still has its limitations ${ }^{[17]}$. In addition to smaller absolute survival benefits in patients with macrovascular invasion and/or extrahepatic spread, drug availability and its costs are the major challenges for its use especially, in developing countries as Egypt. So, the role of sorafenib in advanced HCC should be conformed, and additional trials of other possible systemic chemotherapeutic agents are also needed, especially after the promising results of some other chemotherapeutic regimens ${ }^{[18-20]}$. The role of systemic chemotherapy in the treatment of advanced HCC was evaluated and reviewed in many studies ${ }^{[17,21]}$. In this study, safety and efficacy of low dose of doxorubicin and 5 FU along with supportive treatment were evaluated compared to best standard of supportive care alone in patients with advanced $\mathrm{HCC}$, the overall response was $16.7 \%$ (PR) with disease control of $70 \%$ (16.7\% PR, 53,3\% SD and 0\% CR) in treatment arm compared with $0 \%$ OARin control arm, this results differ from the results achieved by previous studies e.g. Yeo et $a l^{[11]}$ who studied doxorubicin versus (PIAF) combination chemotherapy in patients with HCC carcinoma, where the OAR was $20.9 \%$ in the treatment arm, and Qin et al ${ }^{[22]}$ who studied the efficacy and survival 
benefits of FOFOX 4 compared to doxorubicin in patients had advanced HCC, where the RR was $8.15 \%$ in FOLFOX4 arm, this difference may be due to small number of the patients in our study, all patients were Child class A and most patients had received primary treatment for localized disease before enrollment in this study, but in another study conducted in Egypt by Farrag $\mathrm{A}^{[18]}$ evaluating the role of metronomic dose of capecitabine in patients with advanced HCC, the RR was $16 \%$ and disease control was $69 \%$, nearly the same results obtained in our study, however, our protocol less expensive and more compliant with the patients. Generally, regarding RR, the results obtained in this study are comparable to or slightly better than the results obtained in other studies evaluating old and newer chemotherapeutic agents in the treatment of advanced HCC. There were two small randomized controlled trials studied chemotherapy versus best supportive care, one tested the efficacy of single agent doxorubicin and another tested enteric- coated tegafur/ uracil, where median survival was 2.7 and 12.1 months in two studies versus 1.9 and 6.2 in best supportive care respectively ${ }^{[10,23]}$. Nolatrexede, a thymidylate synthetase inhibitor was evaluated in phase III randomized controlled trial compared to doxorubicin to determine overall survival benefits in patients with advanced hepatocellular carcinoma: RR was $1.4 \%$ and $4.0 \%$ favoring doxorubicin ${ }^{[12]}$. Also, other agents e.g. gemcitabine, taxanes, capecitabine, and cisplatin were studied in the treatment of advanced HCC, its results were comparable or slightly inferior to our results ${ }^{[24-29]}$. Regarding overall survival (OS) and progression free survival (PFS), in our study, the median OS was 8 months in treatment arm compared to 6 months in control arm while PFS was 5 months with 6 months PFS rate $10 \%$ and 3.5 months with 6 months PFS rate $3.3 \%$ in treatment arm and control arm respectively. These results were comparable with other obtained in previous studies mentioned above. Compared with the results obtained in SHARP trial ${ }^{[7]}$ evaluating the role of sorafenib in advanced HCC compared to placebo, in which the median OS was 10.7 months compared to 7.9 months in sorafenib and placebo groups respectively, and time to symptomatic tumor progression was 4.1 versus 4.9 favoring placebo, as shown, the results in our study differ from that of SHARP study, this difference may be due to small number in our study and different inclusion criteria in both trials. The toxicity profile of this regimen was very low regarding hamatological and non- haematological toxicities, so, the drugs were tolerated and convenient with the patients.

\section{Conclusion}

Aadvanced HCC remains a challenging disease with bad prognosis, the median survival in most studies ranging from 6 to 9 months with response rates $10-15 \%$ to chemotherapeutic agents. Because of expense and some unresolved issues regarding sorafenib optimal use in Egyptian patients where prevalence of HCC involves a large sector of these populations, a substantial needs for more effective treatment options still present. Based on the results of this study compared with other obtained in previous trials regarding overall survival, progression free survival, response rates, and safety profile, this protocol may confer some benefits for patients with advanced HCC and may provide another profitable treatment option.

\section{References}

1. Torre, L.A., Bray, F., Siegel, R.L., et al. Global canncer statistics, 2012. (2015) CA Cancer J Clin 65(2): 87-108.

2. Venook, A.P., Papandreou, C., Furuse, J., et al. The incidence and epidemiology of hepatocellular carcinoma: a global and regional perspective. (2010)Oncologist 15(4): 5-13.

3. Ibrahim, A.S., Khaled, H.M., Mikhail, N.H., et al . Cancer Incidence in Egypt: Results of the National Population-Based Cancer Registry Program. (2014) J Can Epid 2014 (2014).

4. Tinkle, C.L., Haas-Kogan, D. Hepatocellular carcinoma: natural history, current management, and emerging tools. (2012) Biologics 6: 207-219.

5. Bruix, J., Sherman, M., Llovet, J.M., et al. Clinical management of hepatocellular carcinoma: Conclusions of the Barcelona-2000 EASL conference. European Association for the Study of the Liver. (2001) J Hepatol 35(3): 421-430.

6. Kudo, M., Matsui, O., Izumi, N., et al. JSH Consensus-based clinical practice guidelines for themanagement of hepatocellular carcinoma: 2014 Update by the liver cancer study group of Japan. (2014) Liv Can 3(3-4): 458-468

7. Llovet, J.M., Ricci, S., Mazzaferro, V., et al. Sorafenib in advanced hepatocellular carcinoma. (2008) N Engl J Med 359(4): 378-390.

8. Germano, D., Daniele, B. Systemic therapy of hepatocellular carcinoma: Current status and future perspectives. (2014) World J Gastroenterol 20(12): 3087-3099.

9. Nerenstone, S.R., Ihde, D.C., Friedman, M.A. Clinical trials in primary hepatocellular carcinoma: Current status and future directions. (1988) Cancer Treat Rev 15(1): 1-31.

10. Lai, C.L., Wu, P.C., Chan, G.C., et al. Doxorubicin versus no antitumor therapy in inoperable hepatocellular carcinoma. A prospective randomized trial. (1988) Cancer 62(3): 479-483.

11. Yeo, W., Mok, T.S., Zee, B., et al. A randomized phase III study of doxorubicin versus cisplatin/interferon alpha-2b/doxorubicin/fluorouracil (PIAF) combination chemotherapy for unresectable hepatocellular carcinoma. (2005) J Natl Can Inst 97(20): 1532-1538.

12. Gish, R.G., Porta, C., Lazar, L., et al. Phase III randomizedcontrolled trial comparing the survival of patients with unresectable hepatocellularcarcinoma treated with nolatrexed or doxorubicin. (2007) J ClinOncol 25(21): 3069-3075.

13. Tetef, M., Doroshow, J., Akman, S., et al. 5-Fluorouracil and highdose calcium leucovorin for hepatocellular carcinoma: aphase II trial. (1995) Can Invest 13(5): 460-463.

14. Link, J.S., Bateman, J.R., Paroly, W.S., et al. 5-Fluorouracil inhepatocellular carcinoma: Report of twenty-one cases. (1977) Cancer 39(5): 1936-1939.

15. Porta, C., Moroni, M., Nastasi, G., et al. 5-Fluorouracil andd, 1-leucovorin calcium are active to treat unresectable hepatocellular carcinoma patients: preliminary results of aphase II study. (1995) Oncology 52(6): 487-491.

16. Kevin, S.B. Chemotherapy and Other SystemicTherapies for Hepatocellular Carcinoma and Liver Metastases. (2006) Semin Interv Radiol 23(1): 99-108.

17. Abdel-Rahman, O. Systemic therapy for hepatocellular carcinoma (HCC): from bench to bedside. (2013) J Egypt Natl Can Inst 25(4): $165-171$.

18. Farrag, A. Efficacy and Toxicity of Metronomic Capecitabine in Advanced Hepatocellular Carcinoma. (2012) J Can Ther 2012(3): 71-77. 19. Nakamura, M., Nagano, H., Marubashi S, et al. Pilot Study of Combination Chemotherapy of S-1, a Novel Oral DPD Inhibitor, and Interferon- $\alpha$ for Advanced Hepato- cellular Carcinoma with Extrahepatic Metastasis. (2008) Cancer 112(8): 1765-1771.

20. Obi, S., Yoshida, H., Toune, R., et al. Combination Therapy of Intra-arterial 5-Fluorouracil and Systemic Inter- feron-Alpha for Advanced Hepatocellular Carcinoma with Portal Venous Invasion. (2006) Cancer 106(9): 1990-1997. 
21. Asghar, U., Meyer, T. Are there opportunities for chemotherapy in the treatment of hepatocellular cancer? (2012) J Hepatol 56(3): 686695.

22. Qin, S., Bai, Y., Lim, H.Y., et al. Randomized, Multicenter, Open-Label Study of OxaliplatinPlus Fluorouracil/Leucovorin Versus Doxorubicin As Palliative Chemotherapy in Patients With AdvancedHepatocellular Carcinoma From Asia. (2013) J clinoncol 31(28): 3501-3508. 23. Ishikawa, T., Ichida, T., Sugitani, S., et al. Improved survival with oral administration of enteric-coated tegafur/uracilfor advanced stage IV-A hepatocellular carcinoma. (2001) J Gastroenterol Hepatol 16(4): 452-459.

24. Chao, Y., Chan, W.K., Birkhofer, M.J., et al. Phase II and pharmacokinetic study of paclitaxel therapy for unresectable hepatocellular carcinoma patients. (1998) Br J Cancer 78(1): 34-39.

25. Hebbar, M., Ernst, O.,Cattan, S., et al. Phase II trial of docetaxel therapy in patients with advanced hepatocellular carcinoma. (2006) Oncology 70(2): 154-158.

26. Yang, T.S., Wang, C.H., Hsieh, R.K., et al. Gemcitabine and doxorubicin for the treatment of patients with advanced hepatocellular carcinoma: a phase I-II trial. (2002) Ann Oncol 13(11): 1771-1778.

27. Guan, Z., Wang, Y., Maoleekoonpairoj, S., et al. Prospective randomised phase II study of gemcitabine at standard or fixed dose rate schedule in unresectable hepatocellular carcinoma. (2003) Br J Cancer 89(10): 1865-1869.

28. Louafi, S., Boige, V., Ducreux, M., et al. Gemcitabine plus oxaliplatin (GEMOX) in patients with advanced hepatocellular carcinoma (HCC): results of a phase II study. (2007) Cancer 109(7): 1384-1390.

29. Park, S.H., Lee, Y., Han, S.H., et al. Systemic chemotherapy with doxorubicin, cisplatin and capecitabine for metastatic hepatocellular carcinoma. (2006) BMC Cancer 6: 3. 